\section{Senkt Sport die Sterberate bei Krebs?}

Onkologen sind sich darin einig, dass Sport auch für Krebspatienten gut sein kann. US-Ärzte werteten jetzt eine Vielzahl von Studien aus, um genauer sagen zu können, wer am meisten wie von körperlicher Aktivität profitiert.

W issenschaftler des Nationalen Krebsinstituts analysierten insgesamt 45 Publikationen zum Thema „Sport bei Krebspatienten“. Randomisierte kontrollierte Studien zu körperlicher Aktivität und Insulinspiegeln, Entzündungsprozessen und Immunabwehr sind sehr heterogen, sodass sich daraus noch keine konkreten Empfehlungen ableiten lassen. Die besten Daten zur Assoziation zwischen körperlicher Aktivität und Sterberate liefern derzeit Beobachtungsstudien mit Brustkrebspatientinnen. In den meisten Studien geht körperliche Aktivität mit einer Verringerung der krebsspezifischen sowie der Gesamtsterberate einher. Bei sechs Studien wurde die Brustkrebs-Sterberate signifikant um 41-51\% reduziert.

Eine ähnlich starke Reduktion wie bei Brustkrebs fanden die Wissenschaftler in Beobachtungsstudien bei Patienten mit Kolorektalkarzinom (41-61\%). Deshalb wird dieser Zusammenhang derzeit in der ersten randomisierten kontrollierten Interventionsstudie überprüft (CHALLENGE: The Colon Health and Life Long Exercise Change): 963 Darmkrebspatienten im Stadium II oder III der Erkrankung machen mit und ohne Anleitung nach einem strukturierten Programm körperliche Übungen und erhalten allgemeine Informationen über einen gesunden Lebensstil. Die Kontrollgruppe erhält nur die Informationen.

Ziel der Intervention ist, die Patienten soweit zu trainieren, dass sie ihre Leistungsfähigkeit von 10 MET-Stunden (Metabolisches Äquivalent) auf 27 METStunden pro Woche steigern. Das entspricht etwa einer Stunde täglichen strammen Gehens oder einer Stunde

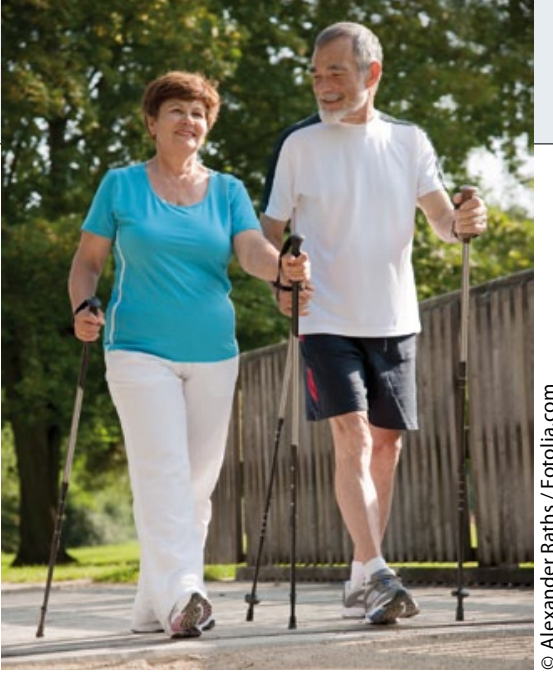

Krebspatienten, die körperlich aktiv sind, leben möglicherweise länger.

täglichen Joggens an dreieinhalb Tagen in der Woche.

Fazit: Sport kann bei Krebskranken die Lebensqualität verbessern und nach erfolgreicher Therapie das Rezidivrisiko senken. Den Ergebnissen von 27 Studien zufolge ist regelmäßige körperliche Aktivität wohl auch mit einem verringerten Sterberisiko bei Brust- und Darmkrebs assoziiert.

Peter Leiner

Ballard-Barbash R et al. Physical activity, biomarkers, and disease outcomes in cancer survivors: a systematic review. J Natl Cancer Inst. 2012;104(11):815-40.

\title{
Schmerzlinderung im Auge des Betrachters
}

Zwei Punkte runter auf der Schmerzskala - das gilt als Erfolg in der Schmerztherapie. Doch bei intensiven Schmerzen kann dies immer noch erhebliches Leid bedeuten. Wäre es nicht besser, die Patienten danach zu fragen, welche Schmerzlinderung sie erreichen wollen: Was ist ihr "Personalized Pain Goal"?

tudien zufolge korreliert die Zufrie$S$ denheit von Patienten mit ihrem Schmerzmanagement nicht unbedingt mit der berichteten Schmerzintensität oder der Schmerzlinderung. Vielmehr hängt sie stark davon ab, ob sie sich eingebunden fühlen und wie gut die Kommunikation mit den Behandlern ist.

Das Prinzip des persönlichen Schmerzziels („Personalized Pain Goal, PPG) wird deshalb von manchen Ärzten favorisiert. Hier geht es um die eigenen Kriterien des Patienten. Er beschreibt, Schmerzen welcher Intensität auf einer Skala von $0-10$ es ihm seiner Ansicht noch ermöglichen würden, sich physisch, funktionell und psychosozial wohlzufühlen. Wie stabil das PPG ist und wie es sich mit den klinischen Schmerzkriterien vergleichen lässt, untersuchte die Sup- portive Care Clinic an der Universität Texas. Dort wird das Prinzipt des PPG seit einiger Zeit anwendet.

Für die Studie wurden Berichte von 465 konsekutiven Krebspatienten ausgewertet. Dabei wurde eine klinische Schmerztherapie dann als erfolgreich gewertet, wenn sie die Schmerzen um mindestens $30 \%$ senkte oder um mindestens zwei Punkte auf der Schmerzskala von 0 bis 10 . Erfolg beim PPG bedeutete es hingegen, wenn der Schmerz infolge der Behandlung auf den gewünschten Level oder darunter sank.

Vor der Behandlung litten 152 (34\%) der Patienten unter milden (1-4 Punkte auf der Skala von 0-10), 95 (21\%) unter moderaten (5-6) und 163 (37\%) unter schweren Schmerzen (7-10). Das mittlere PPG der Patienten lag während der ersten Befragung bei 3, unabhängig vom ursprünglichen Schweregrad der Schmerzen. Das Ziel blieb nach einem Follow-up von 14 Tagen stabil.

Klinisch sprachen die Patienten mit schweren Schmerzen häufiger an $(60 \%)$ als solche mit moderaten (40\%) und milden Schmerzen (33\%). Das PPG wurde dagegen häufiger bei Patienten mit milden Schmerzen erreicht (63\%) als bei solchen mit moderaten (44\%) oder schweren Schmerzen (27\%).

Fazit: Die Mehrheit der Patienten war in der Lage, ihren erwünschten Level der Schmerzlinderung anzugeben. Das mittlere PPG betrug dabei 3 und erwies sich als sehr stabil. $53 \%$ der Patienten erreichten ihr PPG allerdings nicht. Obwohl ihre Schmerzen in der Klinik erfolgreich gelindert wurden, war das Ergebnis für die Mehrzahl der Patienten schlicht nicht gut genug. Christina Berndt

Dalal S et al. Achievement of personalized pain goal in cancer patients referred to a supportive care clinic at a comprehensive cancer center. Cancer. 2012;118(15):3869-77. 